\title{
The effect of short-term intermittent hypoxic exposure on heart rate variability in a sedentary population
}

\author{
CA Lizamore ${ }^{1,2}$, Y Kathiravel ${ }^{3}$, J Elliott ${ }^{4}$, J Hellemans ${ }^{5}$, MJ Hamlin ${ }^{1}$ \\ ${ }^{1}$ Department of Tourism, Sport and Society, Lincoln University, Christchurch, New Zealand. \\ ${ }^{2}$ School of Sport and Physical Education, University of Canterbury, Christchurch, New Zealand \\ ${ }^{3}$ Sports Doctors, Christchurch, New Zealand, \\ ${ }^{4}$ Department of Medicine, University of Otago, Christchurch, New Zealand, \\ ${ }^{5}$ Sportsmed, Christchurch, New Zealand.
}

Received: August 14, 2014

Accepted: November 18, 2015

\begin{abstract}
While the effects of instantaneous, single-bout exposure to hypoxia have been well researched, little is known about the autonomic response during, or as an adaptation to, repeated intermittent hypoxic exposure (IHE) in a sedentary population. Resting heart rate variability (HRV) and exercise capacity was assessed in 16 participants (8 receiving IHE, [Hyp] and 8 receiving a placebo treatment [C]) before and after a 4-week IHE intervention. Heart rate variability was also measured during an IHE session in the last week of the intervention. Post-intervention, the root mean squared successive difference (rMSSD) increased substantially in Hyp (71.6 $\pm 52.5 \%$, mean change $\pm 90 \%$ confidence limits) compared to $\mathrm{C}$ suggesting an increase in vagal outflow. However, aside from a likely decrease in submaximal exercise heart rate in the Hyp group $(-5.0 \pm 6.4 \%)$ there was little evidence of improved exercise capacity. During the week 4 IHE measurement, HRV decreased during the hypoxic exposure (reduced R-R interval: $-7.5 \pm 3.2 \%$; and rMSSD: $-24.7 \pm 17.3 \%$ ) suggesting a decrease in the relative contribution of vagal activity. In summary, while 4 weeks of IHE is unlikely to improve maximal exercise capacity, it may be a useful means of increasing HRV in people unable to exercise.
\end{abstract}

Keywords: autonomic nervous system, sedentary lifestyle, interval hypoxia, simulated altitude, physical fitness, health

Heart rate variability (HRV) is the analysis of the variation in the beat to beat intervals in the heart rhythm, which reflects the autonomic nervous system (ANS) innervation on the sinus node (25). Heart rate variability is also responsive to the changes in the ANS associated with external stimuli such as real or simulated altitude (21). For example, an initially sharp increase in sympathetic activity is observed upon arrival at altitude $(7,28)$, which gradually declines through the acclimatization process $(14,23)$. The reduction in HRV is largely due to a reduction in the parasympathetic drive at the sinus node during hypoxia, the mechanism behind which has been well described by Roche et al. (20). The recovery of HRV to baseline levels upon return to normoxic ambient air depends on the length and severity of the initial exposure. That is, after 12 hours of continuous hypoxic exposure, a pronounced (dampened) effect on HRV is still evident an hour after returning to normoxic ambient air (7), but after a brief hypoxic exposure of $15 \mathrm{~min}$, recovery is almost immediate (20).

Corresponding author: Michael John Hamlin

Department of Tourism, Sport and Society, Lincoln University, Christchurch, New Zealand

Lincoln 7647, PO Box 85084, Christchurch, New Zealand

Phone: +64 3423 0489, Fax: +64 3325 3857; E-mail: mike.hamlin@lincoln.ac.nz 
Simulated altitude procedures are used in both athletes $(2,9)$ and non-athletes $(4,8)$ alike. However, while the acute effects of single or continuous hypoxic exposures have been well-documented, the autonomic response during repeated bouts of intermittent hypoxic exposure (IHE) is currently unknown. Moreover, while IHE has been suggested as a safe means of improving exercise economy (4) and baroreflex sensitivity (8) in participants with cardiovascular disease, little is known about the training effect of IHE on HRV in a sedentary, middle aged population. The aim of this research was to investigate the instantaneous and adaptive effects of 4 weeks of IHE on HRV in a sedentary, middle aged population. As a dampened HRV is associated with poorer coronary health (4), an intervention that serves to increase HRV may have a restorative effect on cardiac health.

\section{Materials and Methods}

\section{Participants}

Inclusion criteria involved engaging in less than $30 \mathrm{~min}$ of physical activity on most days of the week (6), but otherwise generally healthy and aged between 45-60 years. Exclusion criteria included uncontrolled hypertension or cholesteraemia, any condition where peripheral oxygen saturation $\left(\mathrm{SpO}_{2}\right)$ is already compromised, melanoma, pregnancy, or by their own or the screening physician's discretion. Following a health assessment, 16 participants were randomly selected into either a group receiving IHE treatments (Hyp) $(\mathrm{n}=8$; age: $56.5 \pm 5.5$ years; 3 males, 5 females; height: $171.0 \pm 10.1 \mathrm{~cm}$; weight: $82.3 \pm 13.5 \mathrm{~kg}$; body fat: $33.5 \pm$ $4.5 \%$; mean $\pm \mathrm{s})$ or a placebo $(\mathrm{C})(\mathrm{n}=8$; age: $56.1 \pm 5.1$ years; 2 males, 6 females; height: $164.6 \pm 8.4 \mathrm{~cm}$; weight: $81.3 \pm 27.2 \mathrm{~kg}$; body fat: $35.2 \pm 12.9 \%$ ). Participants remained blind to their groups throughout the study. All participants signed a written informed consent prior to participation. This study was approved by the local University Human Ethics Committee.

\section{Baseline and post-intervention testing}

Quantification of physical activity at baseline and post intervention was completed using the International Physical Activity Questionnaire, long-format (IPAQ-LF), and a 4-day diary detailing nutritional intake, sleep, mood, medication and physical activity. Participants were asked to replicate these on subsequent testing days. At baseline, participants were familiarised with the testing equipment and procedures, and anthropometric data was collected including body composition and weight (InBody230, Biospace Co. Ltd., Seoul, South Korea), height and age.

\section{Resting heart rate, heart rate variabilty, and systolic blood pressure}

During the last 5 minutes of a 10-minute resting period, heart rate (HR), HRV and systolic blood pressure (SBP) were measured. Heart rate variability was detected using a heart rate monitor (RS800CX, Polar Electro Oy, Kempele, Finland) and belt (Wearlink W.I.N.D, Polar Electro Oy, Kempele, Finland) and analysed using Polar software (Polar ProTrainer 5, Version 5.41.000). To measure heart rate variability, the root mean square of successive differences in the RR interval (rMSSD) and RR interval were used to provide an indication of vagal outflow. These measures are more resistant to breathing artefact than alternative analyses (18). However, to provide a more complete dataset, and to enable greater comparability with other research, the standard deviation of N-N intervals (SDNN) and high frequency (HF: $0.15-0.4 \mathrm{~Hz}$ ) measurements were also reported. 
As HRV can have low repeatability (27) all data were filtered for ectopic beats using the Polar ProTrainer 5 (PPT5) filters (using default settings of moderate filter power and minimum protection zone of $6 \mathrm{bpm}$ ). Data were then visually inspected and any remaining 'spikes' in the data were removed and replaced with the next appropriate RR interval. The detection of RR intervals using similar Polar heart rate technology has been validated against 12-lead ECGs (19) however, there is uncertainty regarding the ability of the PPT5's error correction function to detect non-sinus originating beats which can result in inflated results (29). Therefore, all data were screened for outliers using the Median Absolute Deviation (30), and participants with greater or less than $3 \times$ the population standard deviation as estimated by the median (17) were excluded from the data. Two participants in the hypoxic group (Hyp) were excluded from the resting HRV analyses due to equipment malfunction $(\mathrm{n}=1)$, and an rMSSD outlier ( $\mathrm{n}=1$, rMSSD was $101.9 \mathrm{~ms}$ at baseline). The natural logarithm of the rMSSD (18) was calculated to further reduce the impact of spontaneous respiration, and to improve the accuracy of the measurements.

\section{Blood pressure}

Blood pressure was measured continuously throughout rest and exercise using plethysmography to detect brachial arterial pressure in the finger (MLE1054-V Finometer MIDI, Finapres Medical Systems, The Netherlands). Data were recorded using BeatScope Easy ${ }^{\circledR}$ software (Finapres Medical Systems, The Netherlands).

\section{Oxygen consumption during rest and exercise}

Respiratory gases were collected through a facemask that allowed simultaneous respiration via the nose and mouth (Hans Rudolph, Kansas City, MO, USA), and measured using a breath-by-breath gas analysis system (MetaMax ${ }^{\circledR} 3 \mathrm{~B}$; Cortex Biophysik, Leipzig, Germany). Prior to each battery of tests, the gas analyser was calibrated against $15 \%$ oxygen and $5 \%$ carbon dioxide. Volume was calibrated daily (Hans Rudolph 55303 L syringe; Kansas City, MO, USA).

A modified submaximal Astrand protocol (24) was used to measure submaximal oxygen uptake $\left(\dot{\mathrm{VO}}_{2 \text { submax }}\right)$ and to predict $\dot{\mathrm{V}} \mathrm{O}_{2 \text { peak }}$. Participants started pedalling at $50 \mathrm{rpm}$ on a cycle ergometer starting at a workload of 25 Watts (W) (Monark Ergomedic 818E, Sweden). Resistance increased by $25 \mathrm{~W}$ every 2 min until the participant reached $70 \%$ of their age predicted maximum heart rate $((220-$ age $) * 0.7)(24)$. Participants then continued to cycle at the same workload for an additional $2 \mathrm{~min}$, and then until steady state $(\leq 5 \mathrm{bpm}$ between consecutive min) was achieved.

Breath-by-breath data was exported to an Excel spreadsheet for visual inspection and any outlying data points were removed. The last $5 \mathrm{~min}$ of the $10 \mathrm{~min}$ resting interval, and the last $30 \mathrm{~s}$ of the $2 \mathrm{~min} 50 \mathrm{~W}$ exercise stage were selected, averaged and reported as oxygen consumption per min, per $\mathrm{kg}$ of body mass $\left(\mathrm{ml} \cdot \mathrm{min}^{-1} \cdot \mathrm{kg}^{-1}\right)$ for resting and $\dot{\mathrm{V}} \mathrm{O}_{2 \text { submax }}$ measures, respectively. Heart rate and RPE were measured at the end of the $50 \mathrm{~W}$ stage and used to indicate submaximal heart rate $\left(\mathrm{HR}_{\text {submax }}\right)$ and rating of perceived exertion (RPE50W), respectively. The mean of the $70 \%$ age-predicted steady state HR recordings and the final workload were used to predict $\dot{\mathrm{V}} \mathrm{O}_{2 \text { peak }}$ using the Astrand-Rhyming nomogram and age correction factor. Steady-state heart rates of $\leq 112 \mathrm{bpm}$ were excluded from the analysis as 
their predicted $\dot{\mathrm{V}} \mathrm{O}_{2 \text { peak }}$ could not be accurately measured using the Astrand nomogram. Time domain analyses of HRV can be unreliable during exercise (22), therefore no exercise-related HRV was analysed.

\section{Intermittent hypoxic exposure}

During the 4-week intervention, participants received six 5-min intervals of hypoxic (or placebo) air delivered through a face mask (QuadraLite Facemask, Intersurgical, Intermed, Auckland, New Zealand) interspersed with six 5-min periods of breathing normoxic ambient air, 4 times per week during seated rest. To improve the single blind nature of the study and prevent the participants from guessing whether they were in the test or control group, a progressive decrease in the fraction of inspired oxygen $\left(\mathrm{F}_{\mathrm{i}} \mathrm{O}_{2}\right)$ was used in the test group. Hypoxic air (Compressor Twin, GO2Altitude, Biomedtech, Victoria, Australia) was controlled such that: Hyp: week 1: $\mathrm{SpO}_{2}=95 \%\left(\mathrm{~F}_{\mathrm{i}} \mathrm{O}_{2} \sim 0.21\right)$; week 2: 90\% $\left(\mathrm{F}_{\mathrm{i}} \mathrm{O}_{2} \sim 0.16\right)$; week 3: 85\% $\left(\mathrm{F}_{\mathrm{i}} \mathrm{O}_{2} \sim 0.13\right)$; and week 4: 80-85\% $\left(\mathrm{F}_{\mathrm{i}} \mathrm{O}_{2} \sim 0.10-0.12\right)$; $\mathrm{C}: \mathrm{SpO}_{2}>95 \%$ in week 1-4. Peripheral oxygen saturation was monitored using finger pulse oximetry (Nonin Pulse Oximeter, Plymouth, Minnesota). During week 4, HRV and HR were recorded during each participant's intervention session to ascertain instantaneous ANS response to a full IHE (or placebo) session. Heart rate and HRV measured during the last 2 min of each 5 min interval were extracted and averaged. The average HR and HRV during the hypoxic and placebo air intervals were then compared. See Fig. 1 for an outline of the testing protocol.

Throughout the intervention period participants maintained a diary to record any intervention-related comments. Similar themes in the comments were identified and the total number of referrals to the theme were counted, as was the greatest contribution made by one participant. This was done to identify where repetitive comments by one participant could have skewed the data.

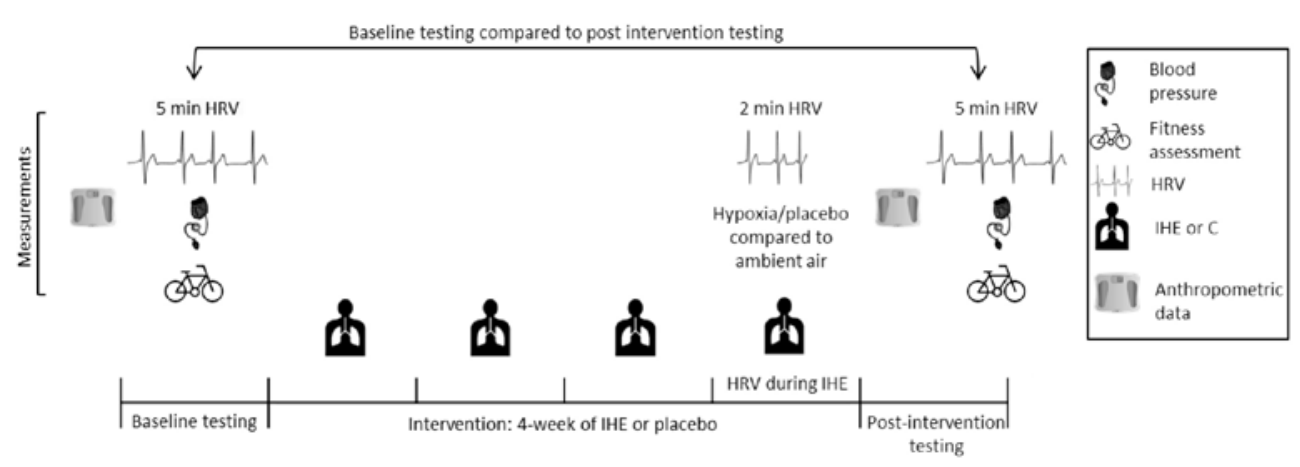

Fig. 1. Testing protocol before, during and after the intervention period.

\section{Statistical analysis}

Statistical analysis was performed using a pre-post parallel groups spreadsheet designed to compute the magnitude of the smallest worthwhile change between groups using a $90 \%$ confidence interval (12). The smallest worthwhile change was determined using Cohen's 
value of 0.2 of the mean difference divided by the between-subject standard deviation. The confidence interval indicates the range of uncertainty of the true value. For a mechanistic inference (in the case of IPAQ-LF, HRV and HR) if the confidence interval overlaps both substantially increased and substantially decreased values, the effect is 'unclear'. In all other cases, the outcome is 'clear' and a statement based on the percent chance of the outcome is given (increased/trivial/decreased). Probabilistic inferences for the qualitative outcome were assigned using the following criteria: $<0.5 \%=$ most unlikely; $0.5-5 \%=$ very unlikely; $5-25 \%$ $=$ unlikely; $25-75 \%=$ possibly $; 75-95 \%=$ likely, $95-99.5 \%=$ very likely, $>99.5 \%=$ most likely (13).

For variables directly affecting health or performance (exercise economy, SBP and predicted $\dot{\mathrm{V}_{2} \text { peak }}$ ), a clinical analysis was performed. In this analysis, if the potential for benefit was acceptable but the risk of harm was too high, the effect is 'unclear'. An odds ratio of benefit: harm was accepted if the ratio was $<66 \%$. In the case of a clear outcome, a statement of the likelihood that the changes were beneficial/trivial/harmful was made. In this way, the risk of making a Type 1 clinical error (using a harmful effect) is prioritised over a Type 2 error (not using a beneficial effect).

Data was log transformed prior to analysis to reduce non-uniformity of error and displayed as a percent. Baseline values were used as a covariate in all analyses to avoid the effect of regression to the mean, and bias associated with uneven baseline averages (12). All results are expressed as mean change $(\%) \pm 90 \%$ confidence interval. Unless reported in Table I, this is followed by the percent chances that the change has substantially increased/ is trivial/ has substantially decreased (in the case of the mechanistic analyses) or is beneficial/ trivial/ harmful (in the case of clinical analyses). Finally, the qualitative outcome is reported.

\section{Results}

Raw data are presented in Table I. Although continuous blood pressure was measured, the average over the last 5 minutes of the 10 minute rest period was used as noise and artefact in the continuous recording impacted the reliability of the data.

As a result of 4 weeks of IHE, resting HR in the Hyp group decreased compared to the C group ( $-10.4 \pm 8.7 \%)$. Resting HRV increased substantially in the Hyp group compared to the C group (rMSSD: $71.6 \pm 52.5 \%$ see Fig 2, RR-interval: $7.8 \pm 8.1 \%$, HF: $241.7 \pm 143 \%$ ) following the 4 weeks of IHE treatment. However, there were no clear changes in SDNN $(11.8 \pm 41.9 \%)$, SBP $(-3.4 \pm 8.4 \%)$, or resting oxygen consumption $\left(\dot{\mathrm{VO}}_{2 \text { rest }}:-0.7 \pm 16.9 \%\right)$ between groups.

One participant in the Hyp group was excluded from all exercising oxygen consumption measurements due to missing post-intervention data. All participants completed the $50 \mathrm{~W}$ submaximal stage. There were no clear changes in $\dot{\mathrm{VO}}_{\text {2submax }}$ or RPE50 W between groups at a $50 \mathrm{~W}$ workload $\left(\dot{\mathrm{VO}}_{2 \text { submax }}:-0.1 \pm 13.9 \%\right.$; RPE50 W: $\left.-5.0 \pm 13.0 \%\right)$, however, the Hyp group demonstrated a likely decrease in $\mathrm{HR}_{\text {submax }}(-5.0 \pm 6.4 \%)$ compared to the $\mathrm{C}$ group. Two participants in each group were excluded from the peak oxygen uptake $\left(\dot{\mathrm{V}} \mathrm{O}_{\text {2peak }}\right)$ predictions as their steady state HR was less than $112 \mathrm{bpm}$. Changes in estimated $\dot{\mathrm{VO}}_{\text {2peak }}$ between groups as a result of IHE training were trivial $(0.8 \pm 7.0 \%)$. 
Table I. Baseline and post intervention resting and exercising mechanistic and clinical variables

\begin{tabular}{|c|c|c|c|c|c|}
\hline & Hyp pre & Hyp post & C pre & C post & $\begin{array}{l}\text { Qualitative outcome } \\
\text { (\% chance of increase, } \\
\text { trivial, or decreased } \\
\text { compared to C) }\end{array}$ \\
\hline \multicolumn{6}{|c|}{ Rest } \\
\hline \multicolumn{6}{|c|}{ Mechanistic variables } \\
\hline $\begin{array}{l}\text { HR } \\
\left(\text { beats } \cdot \min ^{-1} \text { ) }\right.\end{array}$ & $59.2 \pm 7.4$ & $57.8 \pm 6.2$ & $64.38 \pm 9.8$ & $67.4 \pm 8.2$ & Likely decreased (1/6/94) \\
\hline $\begin{array}{l}\text { RR-interval } \\
\text { (ms) }\end{array}$ & $1029 \pm 135$ & $1049 \pm 118$ & $955 \pm 164$ & $921 \pm 116$ & Likely increased $(83 / 16 / 2)$ \\
\hline $\mathrm{rMSSD}(\mathrm{ms})$ & $30.7 \pm 11.9$ & $35.6 \pm 8.1$ & $23.1 \pm 10.9$ & $21.8 \pm 11.0$ & Very likely increased $(95 / 3 / 1)$ \\
\hline $\mathrm{HF}(\mathrm{Hz})$ & $402.7 \pm 302.6$ & $470.6 \pm 217.2$ & $234.1 \pm 150.7$ & $235.7 \pm 255.4$ & Very likely increased $(97 / 2 / 1)$ \\
\hline SDNN & $39.2 \pm 11$ & $45.0 \pm 14.5$ & $29.8 \pm 11.0$ & $35.1 \pm 15.9$ & Unclear (56/27/17) \\
\hline \multicolumn{6}{|c|}{ Clinical variables } \\
\hline $\mathrm{SBP}(\mathrm{mmHg})$ & $120.7 \pm 19.5$ & $122.6 \pm 21.0$ & $109.4 \pm 12.7$ & $117.0 \pm 11.8$ & Unclear $(9 / 37 / 54)$ \\
\hline $\begin{array}{l}\dot{\mathrm{VO}_{2}} \\
\left(\mathrm{ml} \cdot \mathrm{min}^{-1} \cdot \mathrm{kg}^{-1}\right)\end{array}$ & $3.1 \pm 1.1$ & $3.3 \pm 0.5$ & $2.9 \pm 0.7$ & $3.4 \pm 0.7$ & Unclear $(24 / 46 / 30)$ \\
\hline \multicolumn{6}{|c|}{ Exercise } \\
\hline $\begin{array}{l}\dot{\mathrm{VO}}{ }_{2 \text { submax }} \\
\left(\mathrm{ml} \cdot \mathrm{min}^{-1} \cdot \mathrm{kg}^{-1}\right)\end{array}$ & $10.0 \pm 1.8$ & $11.0 \pm 1.7$ & $11.3 \pm 1.8$ & $12.1 \pm 2.2$ & Unclear (31/38/32) \\
\hline $\mathrm{HR}_{\text {submax }}$ & $90.3 \pm 8.7$ & $86.3 \pm 7.9$ & $90.8 \pm 14.0$ & $91.0 \pm 12.7$ & Likely decreased $(2 / 23 / 75)$ \\
\hline $\mathrm{RPE}_{50 \mathrm{~W}}$ & $10.5 \pm 1.3$ & $10.0 \pm 1.3$ & $10.0 \pm 1.9$ & $10.3 \pm 1.6$ & Unclear $(12 / 29 / 60)$ \\
\hline $\begin{array}{l}\text { Predicted } \\
\dot{\mathrm{VO}}_{2 \text { peak }} \\
\left(\mathrm{ml} \cdot \mathrm{min}^{-1} \cdot \mathrm{kg}^{-1}\right)\end{array}$ & $23.6 \pm 2.5$ & $23.5 \pm 2.6$ & $25.0 \pm 10.0$ & $24.9 \pm 10.8$ & Likely trivial $(9 / 86 / 5)$ \\
\hline
\end{tabular}

Data are mean $\pm \mathrm{SD}$, Qualitative outcome is expressed as: \% chance of increase/trivial/decrease for mechanistic variables, and \% chances of beneficial/trivial/harmful in clinical variables. Hyp: Hypoxic exposure group; C: control group; Pre: baseline recording; Post: 1-3 days post intervention; HR: Heart rate; RR-interval: Average interval between R to R peaks in the HR trace; rMSSD: Root mean square successive difference; SBP: Resting systolic blood pressure; $\dot{\mathrm{VO}}{ }_{2 \text { rest }}$ : Oxygen uptake during rest; $\dot{\mathrm{VO}}_{2 \text { submax }}$ : oxygen uptake during the last $30 \mathrm{~s}$ of the $50 \mathrm{~W}$ interval; $\mathrm{HR}_{\text {submax }}$ : average heart rate taken at the end of each min during the $50 \mathrm{~W}$ exercise interval; RPE50 $\mathrm{W}$ : average rating of perceived exertion taken that the end of the $50 \mathrm{~W}$ exercise interval; predicted $\dot{\mathrm{VO}}_{2 \text { peak }}:$ peak oxygen uptake predicted using Astrand nomogram

During the week-4 HRV and HR assessments, there was a substantial decrease in rMSSD and the RR-interval, and an increase in HR when participants in the Hyp group went from breathing ambient air to hypoxic air (rMSSD: $30.0 \pm 2.6 \mathrm{~ms}$ to $25.4 \pm 1.8 \mathrm{~ms}$; RR-interval: $976 \pm 20 \mathrm{~ms}$ to $883 \pm 16 \mathrm{~ms}$; HR: $62.4 \pm 1.5 \mathrm{bpm}$ to $68.2 \pm 1.3 \mathrm{bpm}$ ) compared to the $\mathrm{C}$ group who went from breathing placebo to ambient air (rMSSD: $18.2 \pm 1.5 \mathrm{~ms}$ to $20.3 \pm 2.1 \mathrm{~ms}$; RR-interval: $970 \pm 16 \mathrm{~ms}$ to $950 \pm 35 \mathrm{~ms}$; HR: $62.4 \pm 1.2 \mathrm{bpm}$ to $63.6 \pm 2.6 \mathrm{bpm})$. The differences between groups in these variables are as follows: rMSSD: $-24.7 \pm 17.3 \%, 1 / 1 / 98$, very likely decreased; RR-interval: $-7.5 \pm 3.2 \%, 0 / 0 / 100$, most likely decreased; HR: $8.8 \pm$ $3.4 \%, 100 / 0 / 0$, most likely increased (see Fig 3 ). 
Hyp

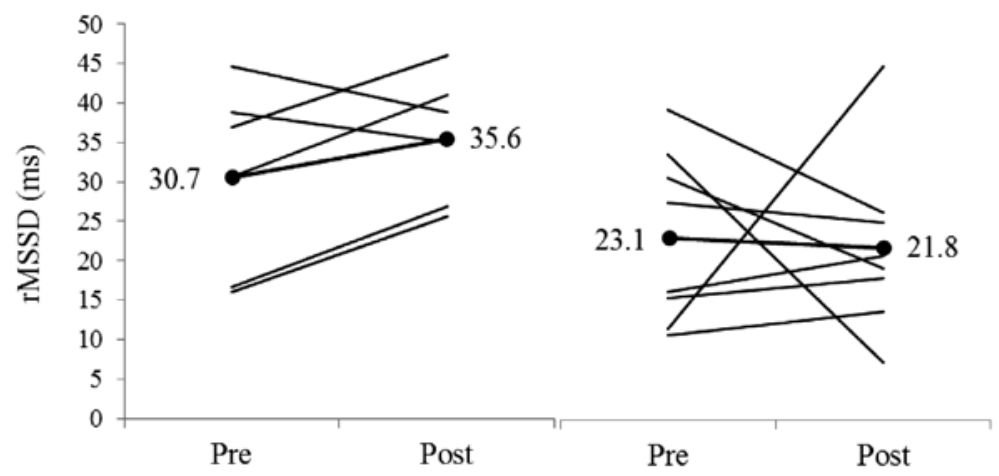

Fig. 2. Root mean square of successive differences (rMSSD) at baseline and post-intervention

Legend: Thin lines indicate individual responses; thicker lines indicate the group average (which are also indicated numerically on either end of the mean line).

Hyp: group receiving hypoxic air during intermittent hypoxic treatments; C: Control Group receiving normoxic (placebo) air during intermittent hypoxic treatment; rMSSD: root mean square successive difference.
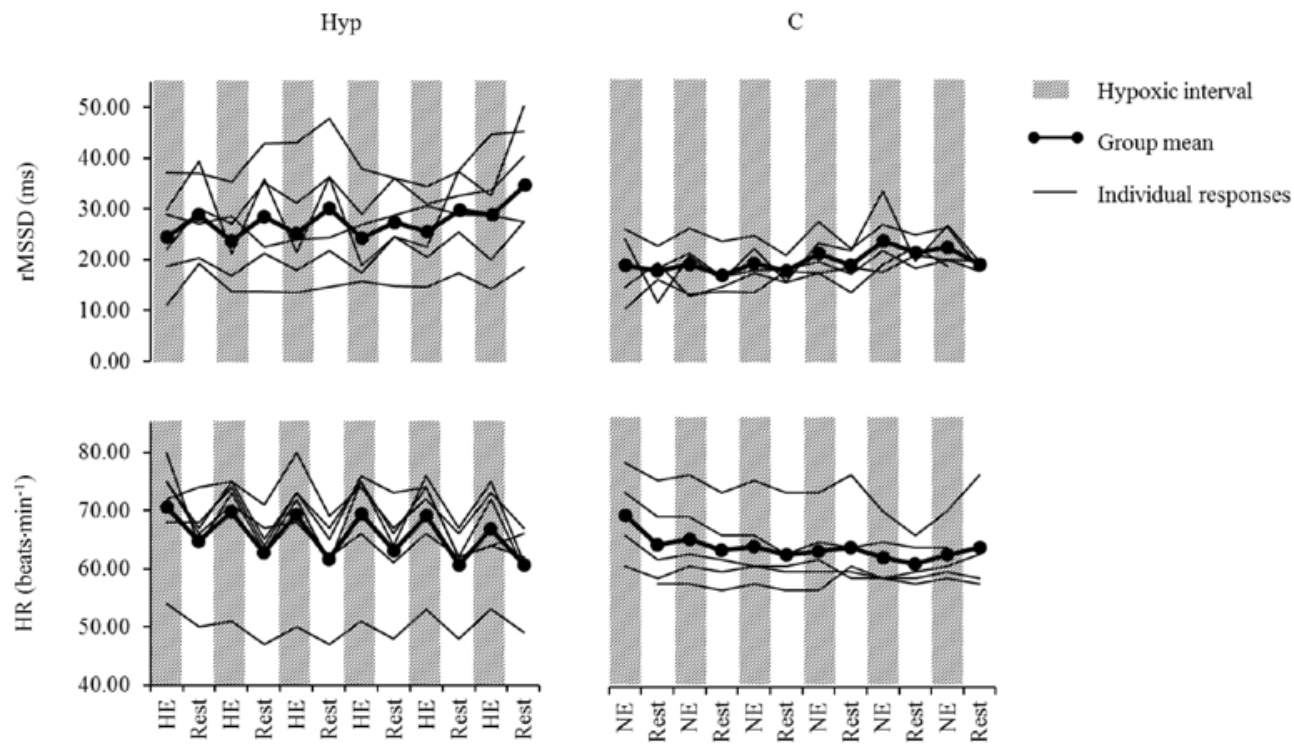

Fig. 3. Heart rate and rMSSD responses during a typical week 4 intermittent hypoxic exposure session

Legend: Hyp: group receiving hypoxic air during intermittent hypoxic treatments; Control: Group receiving normoxic (placebo) air during intermittent hypoxic treatment. rMSSD: Root mean square successive difference;

HR: Heart rate; IHE: Intermittent hypoxic exposure; HE: 5-min hypoxic exposure interval;

Rest: 5-min ambient air interval; NE: 5-min normoxic (placebo) interval. Lined areas indicate the hypoxic or normoxic placebo intervals. Thin lines indicate individual participant results, whereas the thicker lines indicate the group mean. 
The most frequently reported comments relating to the IHE sessions were "fine" and "tired". More participants in Hyp than C reported breathing discomfort (usually regarding an absence of "enough air") or headaches (Fig 4).

There were no clear changes in physical activity (total METS) between groups over the course of the study. Food and activity diaries indicated that participants had not deviated substantially in their day-to-day nutrition, medication or physical activity habits during the study.

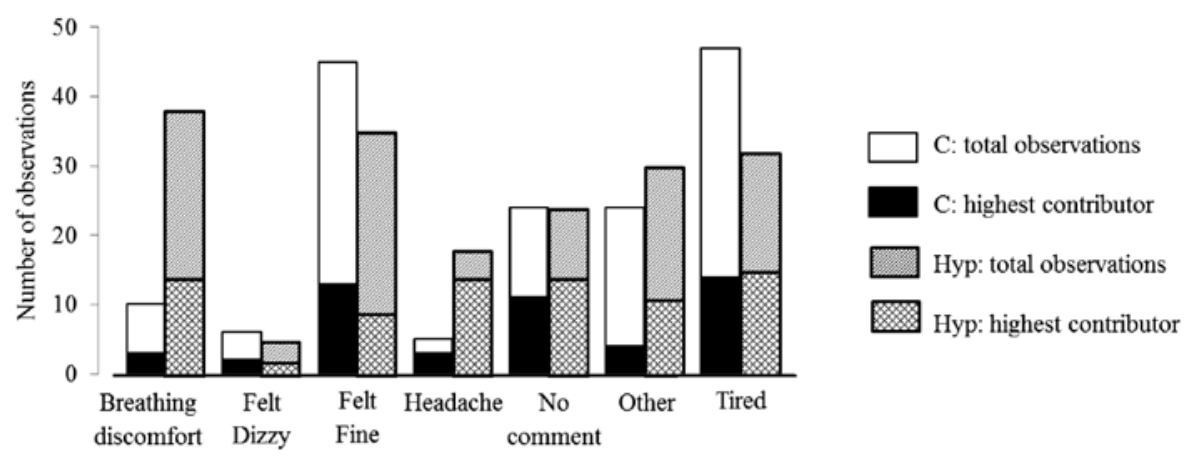

Comment

Fig. 4. Frequencies of recurring comments from participants regarding the hypoxic/normoxic exposure recorded in notebooks over the course of the 4-week intervention period

Legend: Black and white bars on the left represent proportions from the control group, while lined and crossed bars on the right represent proportions from the hypoxic group. As these bars indicate the total number of times the comment was made over the 4-week intervention, irrespective of whether it was from 1 person repeatedly, or many people once, the value of the highest contribution by one person has been indicated (black and crossed sessions, respectively). C: control group receiving normoxic (placebo) air during intermittent hypoxic treatments. Hyp: group receiving hypoxic air during intermittent hypoxic exposure.

\section{Discussion}

The results of this study indicate that although the typical response to breathing hypoxic air is associated with decreased HRV (reflecting increased SNS), the subsequent adaptation following 4 weeks of IHE is associated with increased HRV. To our knowedge, this is the first study to look at the instantaneous and adaptive response of the ANS to repeated hypoxic exposure intervals in a sedentary population.

The overall increase in resting HRV following 4 weeks of IHE is likely an adaptive response to the repeated stress stimulus of the the hypoxic intervals. Indeed, in a similar study in healthy army recruits, 2 weeks of IHE training resulted in reduced vagal withdrawal during the hypoxic exposure suggesting improved tolerance to the hypoxic stress (1). However, no changes in resting HRV were noted in these participants, which could have been due to the short intervention period.

As anticipated, the HF outcomes were very similar to the rMSSD as these two measures are highly correlated to each other. The SDNN measurements reflect both short and longterm components of HRV (26). In this regard, the short 5-min recordings in our study would have limited the capture of long term cyclic components of the HRV and likely contributed to the 'unclear' statistical outcome. 
While we did not detect any improvement in estimated $\dot{\mathrm{V}} \mathrm{O}_{2 \text { peak }}$ or $\dot{\mathrm{V}} \mathrm{O}_{2 \text { submax }}$, we did notice lower HR during submaximal $(50 \mathrm{~W})$ exercise. Interestingly, in addition to improved $\mathrm{HR}_{\text {submax }}$, Burtscher et al. (3) reported significantly lower SBP, HR, and RPE in the group receving IHE following his 3-week intervention. The greater improvement in $\mathrm{VO}_{2 \text { submax }}$ in Burtscher et al. (3) study participants compared to our participants may be attributed to the lower baseline health of the individuals in their study. However, like Burtscher et al. (3), despite the improvement in $\mathrm{HR}_{\text {submax }}$, we did not detect any change in $\dot{\mathrm{VO}}_{2 \text { submax }}$ between the groups, which suggests that IHE seems to improve submaximal HR but has an unclear effect on oxygen efficiency.

As with the resting rMSSD, the decreased $\mathrm{HR}_{\text {submax }}$ may be associated with the increased stress tolerance to the hypoxic exposures yielding reduced vagal withdrawal demonstrated by lower heart rate during physiological stress. Alternatively, haemodynamic adaptation, such as incresed total haemoglobin mass, may account for the reduction in $\mathrm{HR}_{\text {submax }}$. While this has been discovered in similar studies (3), haemodynamic parameters were not measured in this study. However, it would be expected that with improved haemodynamics, improvement in $\dot{\mathrm{VO}}_{2 \text { submax }}$ and $\dot{\mathrm{V}} \mathrm{O}_{2 \text { peak }}$ would result (5). Therefore, in our participants it is likely that the reduced $\mathrm{HR}_{\text {submax }}$ with no apparent change in $\dot{\mathrm{V}} \mathrm{O}_{2 \text { submax }}$ or $\dot{\mathrm{V}} \mathrm{O}_{2 \text { peak }}$ is as a result of improved autonomic stress tolerance and reduced vagal withdrawal during light or moderate exercise.

During the hypoxic intervals, there was a substantial decrease in MSSD, which supports the findings of other researchers $(7,15,16,21)$. What was interesting, however, was the large variation in the individual HRV response to the IHE intervals (see Fig. 3). The measurement of rMSSD reflects vagal modulation which is largely related to the respiratory sinus arrhythmia and therefore can be influenced by breathing frequency (10). Given that there can be substantial individual variation in the hypoxic ventilatory response (11), the change in breathing frequency with hypoxic exposure may be responsible for the decrease in rMSSD, rather than autonomic change. However, while Penttilä et al. (18) did note a decrease in rMSSD with increased breathing frequency, these differences were not statistically significant, and therefore the decrease in rMSSD during hypoxic exposure in our study is likely to predominantly reflect vagal withdrawal, rather than change in respiration.

In addition to the high level of autonomic variation between participants, there was also a wide variation in the nature of the subjective reponses in the participants which were collected in session by session comment books. For example, while most of the participants tolerated the hypoxic intervals well (see "fine" or "no comment" in Fig. 4), a number of the Hyp group detailed mild side effects during the hypoxic interval (such as having an urge to breath faster or more deeply, see "breathing" in Fig. 4). One participant, however, noted headaches, chest pain and blurry vision during the hypoxic exposure interval (note the much higher "highest contributor" portion in the "headache" and "other" bars in Fig. 4). On the advice of the physician, the participant in question stopped the hypoxic interval at the onset of any of these symptoms and only continued with the IHE session following full recovery, and if so desired by the participant. On all occasions, the symptoms were abated following a short return to ambient air and the participant was happy to continue the session. The session was continued at a previsouly well tolerated $\mathrm{F}_{\mathrm{i}} \mathrm{O}_{2}$.

\section{Limitations}

The small sample sizes (Hyp: $\mathrm{n}=8$, and $\mathrm{C}: \mathrm{n}=8$ ), and the necessity to exclude some of the data due to erronous or incomplete datasets increase the risk of Type 1 errors, and reduced the 
extent to which these results can be applied to other populations. Therefore, caution should be used when interpreting these results until the findings can be repeated in a larger population.

Participants were encouraged to be as inclusive as possible when using their comment books, but this notetaking was not monitored. Consequentially, some participants may have recorded data unrelated to the hypoxic exposure, while others neglected to complete the comment books altogether. A focus group debriefing session, or open-ended interview would provide more structured feedback.

In conclusion, four IHE sessions per week for 4 weeks resulted in increased HRV in sedentary, middle-aged adults, and lowered their submaximal exercising HR. However, the IHE intervention did not demonstrate any beneficial effect on aerobic metabolism. If these findings are confirmed in a larger cohort, IHE may prove to be a useful technique for people requiring autonomic stimulation but who are unable to exercise, such as those confined to bed rest or those with cardiovascular disease.

\section{Acknowledgements}

We gratefully acknowledge the New Zealand National Heart Foundation for the stipend provided to the lead researcher in this project. We would also like to express our appreciation to the participants who willingly and cheerfully volunteered their time to this research project. No other funding, grants, or financial support was used for this project.

\section{REFERENCES}

1. Bernardi L, Passino C, Serebrovskaya Z, Serebrovskaya T, Appenzeller O: Respiratory and cardiovascular adaptations to progressive hypoxia. Eur. Heart J. 22, 879-886 (2001)

2. Bonetti DL, Hopkins WG: Sea-level exercise performance following adaptation to hypoxia: a meta-analysis. Sports Med. 39, 107-127 (2009)

3. Burtscher M, Haider T, Domej W, Linser T, Gatterer H, Faulhaber M, Pocecco E, Ehrenburg I, Tkatchuk E, Koch R, Bernardi L: Intermittent hypoxia increases exercise tolerance in patients at risk for or with mild COPD. Respir. Physiol. Neurobiol. 165, 97-103 (2009)

4. Burtsher M, Pachinger O, Ehrenbourg I, Mitterbauer G, Faulhaber M, Puhringer R, Thatchouk E: Intermittent hypoxia increases exercise tolerance in elderly men with and without coronary artery disease. Int. J. Cardiol. 96, 247-254 (2004)

5. Calbet JAL, Lundby C, Koskolou M, Boushel R: Importance of hemoglobin concentration to exercise: Acute manipulations. Respir. Physiol. Neurobiol. 151, 132-140 (2006)

6. Garber CE, Blissmer B, Deschenes MR, Franklin BA, Lamonte MJ, Lee IM, Nieman DC, Swain DP: Quantity and quality of exercise for developing and maintaining cardiorespiratory, musculoskeletal, and neuromotor fitness in apparently healthy adults: Guidance for prescribing exercise. Med. Sci. Sports Exerc. 43, 1334-1359 (2011)

7. Guger C, Krausert S, Domej W, Edlinger G, Tannheimer M: EEG, ECG and oxygen concentration changes from sea level to a simulated altitude of $4000 \mathrm{~m}$ and back to sea level. Neurosci. Lett. 442, 123-127 (2008)

8. Haider T, Casucci G, Linser T, Faulhaber M, Gatterer H, Ott G, Linser A, Ehrenbourg I, Tkatchouk E, Burtscher $\mathrm{M}$, Bernardi L: Interval hypoxic training improves autonomic cardiovascular and respiratory control in patients with mild chronic obstructive pulmonary disease. J. Hypertens. 27, 1648-1654 (2009)

9. Hamlin M, Marshall H, Hellemans J, Ainslie P, Anglem N: Effect of intermittent hypoxic training on $20 \mathrm{~km}$ time trial and $30 \mathrm{~s}$ anaerobic performance. Scand. J. Med. Sci. Sports 20, 651-661 (2010)

10. Hirsch JA, Bishop B: Respiratory sinus arrhythmia in humans: how breathing pattern modulates heart rate. Am. J. Physiol. Heart Circ. Physiol. 241, H620-H629 (1981)

11. Hirshman CA, McCullough RE, Weil J: Normal values for hypoxic and hypercapnic ventilaroty drives in man. J. Appl. Physiol. 38, 1095-1098 (1975) 
12. Hopkins WG: Spreadsheets for analysis of controlled trials, with adjustment for a subject characteristic. Sportscience 10, 46-50 (2006)

13. Hopkins WG, Marshall SW, Batterham AM, Hanin J: Progressive statistics for studies in sports medicine and exercise science. Med. Sci. Sports Exerc. 41, 3-12 (2009)

14. Jun Q, Lan H, Kaixin T, Shiyong Y, Yang Y, Min L: Changes of autonomic nervous system function in healthy young men during initial phase at acute high-altitude exposure. J. Med. Coll. PLA 23, 270-275 (2008)

15. Kanai M, Nishihara F, Shiga T, Shimada H, Saito S: Alterations in autonomic nervous control of heart rate among tourists at 2700 and $3700 \mathrm{~m}$ above sea level. Wilderness Environ. Med. 12, 8-12 (2001)

16. Koelwyn GJ, Wong LE, Kennedy MD, Eves ND: The effect of hypoxia and exercise on heart rate variability, immune response, and orthostatic stress. Scand. J. Med. Sci. Sports 23, e1-e8 (2013)

17. Leys C, Ley C, Klein O, Bernard P, Licata L: Detecting outliers: Do not use standard deviation around the mean, use absolute deviation around the median. J. Exp. Soc. Psychol. 49, 764-766 (2013)

18. Penttilä J, Helminen A, Jartti T, Kuusela T, Huikuri HV, Tulppo MP, Coffeng R, Scheinin H: Time domain, geometrical and frequency domain analysis of cardiac vagal outflow: effects of various respiratory patterns. Clin. Physiol. 21, 365-376 (2001)

19. Porto LGG, Junqueira Jr LF: Comparison of time-domain short-term heart interval variability analysis using a wrist-worn heart rate monitor and the conventional electrocardiogram. Pacing Clin. Electrophysiol. 32, 43-51 (2009)

20. Roche F, Reynaud C, Garet M, Pichot V, Costes F, Barthélémy J-C: Cardiac baroreflex control in humans during and immediately after brief exposure to simulated high altitude. Clin. Physiol. Funct. Imaging 22, 301-306 (2002)

21. Saito S, Tanobe K, Yamada M, Nishihara F: Relationship between arterial oxygen saturation and heart rate variability at high altitudes. Am. J. Emerg. Med. 23, 8-12 (2005)

22. Sandercock GRH, Brodie DA: The use of heart rate variability measures to assess autonomic control during exercise. Scand. J. Med. Sci. Sports 16, 302-313 (2006)

23. Sevre K, Bendz B, Hankø E, Nakstad AR, Hauge A, Kåsin JI, Lefrandt JD, Smit AJ, Eide I, Rostrup M: Reduced autonomic activity during stepwise exposure to high altitude. Acta Physiol. Scand. 173, 409-417 (2001)

24. Siconolfi SF, Cullinane EM, Carleton RA, Thompson PD: Assessing VO2max in epidemiologic studies: modification of the Astrand-Ryhming test. Med. Sci. Sports Exerc. 14, 335-338 (1982)

25. Stein PK, Kleiger RE: Insights from the study of heart rate variability. Ann. Rev. Med. 50, $249-261$ (1999)

26. Task Force of The European Society of Cardiology and The North American Society of Pacing and Electrophysiology: Heart Rate Variability: Standards of measurement, physiological interpretation and clinical use. Eur. Heart J. 17, 354-381 (1996)

27. Tulppo MP, Makikallio TH, Seppanen T, Laukkanen RT, Huikuri HV: Vagal modulation of heart rate during exercise: effects of age and physical fitness. Am. J. Physiol. Heart Circ. Physiol. 274, H424-H429 (1998)

28. Vigo DE, Pérez Lloret S, Videla AJ, Pérez Chada D, Hünicken HM, Mercuri J, Romero R, Nicola Siri LC, Cardinali DP: Heart rate nonlinear dynamics during sudden hypoxia at $8230 \mathrm{~m}$ simulated altitude. Wilderness Environ. Med. 21, 4-10 (2010)

29. Wallén MB, Hasson D, Theorell T, Canlon B, Osika W: Possibilities and limitations of the polar RS800 in measuring heart rate variability at rest. Eur. J. Appl. Physiol. 112, 1153-1165 (2012)

30. Wilcox RR (2010): The normal curve and outlier detection. In: Fundamentals of Modern Statistical Methods: 2nd ed. Springer, New York, pp. 29-45 